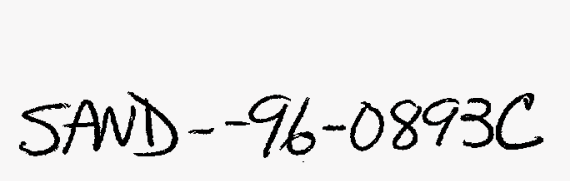

S4N096-0893C

CONF- $960994--5$

Abstract \#198

August 21, 1996

\title{
Monte Carlo Simulations of Ion-Enhanced Island Coarsening
}

Eric Chason ${ }^{a}$ and Bruce K. Kellerman $b$

a Sandia National Laboratories, Albuquerque, NM

b MEMC Electronic Materials, St. Peters, MO

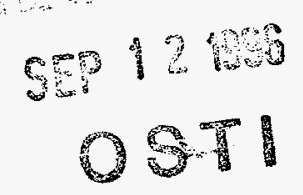

Monte Carlo simulations of growth and ion bombardment have been performed to explore the atomistic processes that occur during ion-assisted growth. The primary elements of the simulation are 1) creation of surface defects (vacancies and adatoms) by ion bombardment and deposition, 2) thermally activated motion of surface defects and 3) recombination of surface vacancies and adatoms. We find that a balance of ion bombardment and deposition (where the creation rate of adatom defects is equal to that of surface vacancies) leads to larger islands and more rapid island coarsening than thermal coarsening with no flux of defects. The presence of the defect flux enhances the breakup of clusters, leading to a broad distribution of island sizes. In comparison, thermal coarsening leads to a more uniform distribution of islands sizes that increases in size much more slowly. Histograms of the evolution of the island size distribution provide a quantitative measure of the ion-induced increase in the rate of coarsening.

\section{Introduction}

Advanced nanostructured materials require well-controlled interface and surface morphologues for multiple applications, e.g., in microelectronics, optoelectronics, X-ray optics and magnetic materials. One method that is being studied for controlling morphology during growth is the use of energetic beams in conjunction with deposition to 


\section{DISCLAIMER}

Portions of this document may be illegible in electronic image products. Images are produced from the best available original document. 
modify surface kinetics and control the growth process. Low energy ion beams have been reported to enhance the smoothness of surfaces during homoepitaxial growth [1] and also to suppress the formation of 3-dimensional islands during heteroepitaxy [2,3]. Although these demonstrations of growth enhancement are encouraging, there is not a good understanding of the atomistic mechanisms behind ion-assisted growth.

In this work, we report on the results of Monte Carlo simulations of ion-assisted growth in which we explore the atomistic mechanisms that lead to growth enhancement. Using properties of ion-induced and growth-induced defects obtained from fundamental studies of surface roughening kinetics, we simulate the response of the surface to sequences of growth and ion bombardment to determine if these mechanisms can reproduce the observed roughening and smoothing behavior. In a previous work, we showed how these mechanisms were consistent with measurements of surface smoothing during homoepitaxy [??] on stepped surfaces where the ion-induced defects enhanced the breakup of small islands and the incorporation of atoms into step edges. By promoting growth by step flow rather than nucleation and coalescence of 2-dimensional islands, a smoother surface morphology was achieved in agreement with RHEED measurements.

In this work, we extend this study to look at the kinetics of island coarsening on singular surfaces in the presence of ion- and growth-induced defect fluxes. We show that the island size during ion assisted processing can be made significantly larger than by purely thermal processes, which in turn can lead to smoother surfaces. However, if the number of ion-induced defects is too large then the continuous nucleation of new islands offsets the enhancement in the island size.

\section{Simulations}

The surface processes considered in the simulations are based on several fundamental studies of low energy ion-induced defects using RHEED (reflection high 
energy electron diffraction). RHEED is extremely sensitive to small amounts of surface roughness, and by measuring the roughening and smoothing kinetics resulting from different combinations of ion bombardment and growth, several features of ion-assisted growth processes have been determined. The number of surface vacancies and adatoms created per ion has been measured for several species of low energy ions [???]. Sequences of growth followed by ion bombardment indicate that the adatoms and surface vacancies can recombine, leading to a smoother surface [???]. Comparison of the temperaturedependent kinetics of surface roughening during ion bombardment with the kinetics of growth roughening indicate that the activation energy for surface vacancy diffusion is comparable to that for adatom diffusion [6]. At elevated temperatures such as those used for ion-assisted growth, recombination of the surface vacancies and adatoms is rapid so that the remaining number of defects after recombination is equal to the sputter yield. Previous simulations also indicate that for the ion energies considered, the sputtering process is random and there is no strong preference for sputtering from low coordinated surface sites [???].

The algorithm for implementing these surface processes in a simulation has been described previously [ DOdson JVST], so it will only be discussed briefly here. The primary steps in the simulation are the creation of defects (surface vacancies and adatoms), the thermally activated hopping of the defects on the surface and the annihilation of defects with each other or with existing clusters. We associate a rate with each of these processes, and events are chosen to be performed on the surface based on their relative rates of occurrence.

The activation energy for surface diffusion of isolated adatoms and surface vacancies is taken to be $0.8 \mathrm{eV}$. An additional $0.2 \mathrm{eV}$ per nearest neighbor is added to the activation energy for hopping of atoms from sites with higher coordination. This extra energy stabilizes clusters and alows nucleation to occur. Vacancy diffusion is implemented by considering the coordination of the final site as well as the intitial site. If 
an atom jumps from a 3-fold coordinated site to a 0 -fold coordinated site, this is a detachment event with an activation energy of $1.4 \mathrm{eV}$. However, if the atom jumps from a 3-fold coordinated site to another 3-fold coordinated site, this is a vacancy jump and the activation energy is $0.8 \mathrm{eV}$. There is no additional energy barrier for hopping between levels on the surface and 4-fold coordinated atoms in the surface are not allowed to move. Annihilation occurs whenever defects of opposite sign occupy the same site by random jumps. There is no attractive interaction between defects of opposite sign. The initial surface is a flat array of $256 \times 256$ atoms and the simulation temperature is $200 \mathrm{C}$. The calculations were performed on an HP Vectra computer with a $133 \mathrm{MHz}$ pentium processor. Typical simulation runs required $6-24 \mathrm{hrs}$ to complete. To obtain sufficient statistics for the island size distributions, 4 - 6 simulation runs under the same conditions were added together.

All the simulations discussed here start with $0.25 \mathrm{ML}$ of deposition at a deposition rate of $0.1 \mathrm{ML} / \mathrm{s}$. After the initial deposition, three different conditions were studied: 1) terminate growth flux, allow thermal coarsening to occur, 2) continue growth flux, turn on equal ion flux (each ion creates one vacancy defect), 3) continue growth flux, turn on equal ion flux (each ion creates 9 defects: 5 vacancies and 4 adatoms). The spatial distribution of the 9 defects was determined from simulations of ion-induced bulk defect creation and diffusion to the surface based on TRIM simulations [ref???]. In all cases, after the initial growth sequence the net defect creation rate (vacancies minus adatoms) is equal to zero so that on average no material is being added to or removed from the surface. However, the total defect creation rate varies from zero (thermal coarsening) to $10 \mathrm{ML} / \mathrm{s}$.

\section{Results}


The surface morphology at different stages of the simulation are shown in figure 1 . Fig. 1a shows the surface after $0.25 \mathrm{ML}$ of growth which is the typical surface morphology before coarsening. The other panels show the surfaces after subsequent $\mathbf{7 7 . 5}$ $s$ of annealing under the following conditions. b) thermal annealing (no growth or ion flux), c) ion bombardment and growth with 1 vacancy defect per ion and d) ion bombardment and growth with 9 defects created per ion. The island sizes in the thermal case appear to be much more regular than for the ion assisted cases. The largest islands are found for the case of ion assisted coarsening with 1 defect/ion (fig. 1c), although there are also many small clusters present. The case of 9 defects /ion (fig. 1d) also has some large clusters, but there is also a large number of small clusters of adatoms and vacancies.

More quantitative understanding can be obtained by looking at the evolution of the island size distributions for the different coarsening conditions (shown in fig. 2). The data are presented as the island size, $\mathrm{n}$, versus the fractional coverage of the surface by islands of size $n$ (equal to the number of islands of size $n$ multiplied by the size of the island and normalized by the area of the surface). In this way, the distribution is weighted so that its magnitude reflects the fraction of the surface covered by islands of size $\mathrm{n}$. Shown in fig 2 are these weighted island size distributions obtained after 17.5, 37.5 and $77.5 \mathrm{~s}$ of processing after growth for the cases of thermal coarsening (a-c), one vacancy per ion ( $d$ f) and 9 defects/ion (g-i). The mean of the weighted distribution is indicated by the vertical dashed line. The evolution of the mean of the distribution with time for each of these cases is shown in fig. 3.

For the case of thermal coarsening, the mean of the weighted island size distribution increases very slowly with time. The distribution also stays quite sharp, consistent with the relatively uniform size of the islands seen in fig. $1 \mathrm{~b}$. The mean of the distribution increases much more quickly in the presence of a simultaneous adatom and vacancy defect flux (fig. $2 \mathrm{~d}-\mathrm{f}$ ) while the width of the distribution becomes very broad. The coverage weighted mean island size at the end of the simulations is much larger for 
the case of 1 vacancy per island than for the case of thermal coarsening (318 compared to 140 atoms).

However, increasing the number of defects created per ion does not lead to a further increase in the mean island size. For the case of 9 defects created per incident ion (fig. $2 \mathrm{~g}-\mathrm{i}$ ), the introduction of multiple defects causes continuous nucleation of new clusters. This shows up as a large peak in the distribution at small island size indicating the continuous presence of many small clusters on the surface. Although the largest islands present are significantly larger than for the thermal case (with clusters as large as 775 atoms present), the creation of small clusters makes the mean of the distribution only slightly larger than for the thermal case.

It is important to recognize that these results do not consider the effects of changing temperature, defect fluxes and activation energies. The addition of athermal defects will not necessarily lead to an increase in the coarsening under all conditions.. The observed effect of ion-enhanced coarsening is caused by changing the balance between multiple surface processes so that the breakup of islands is enhanced without introducing too many new clusters. However, with different values of the simulation parameters the increase in the coarsening rate may not be as significant. For instance, at higher temperatures the detachment rates of atoms from islands may be sufficiently high that the addition of athermal defects would not substantially change the coarsening rate. The significance of the results discussed above is that the addition of athermal defects provide an atomistic mechanism that can lead to an increase in coarsening. Further simulation studies and the development of an analytical theory for ion-assisted coarsening are needed to determine under what range of conditions the increase in coarsening occurs. 


\section{Summary}

We have used Monte Carlo simulations to study the effect of ion-induced defects on the evolution of 2-dimensional island size distributions. A significant increase in the mean island size is observed during simultaneous deposition and ion bombardment when the growth flux is equal to the ion flux and each ion creates one surface vacancy defect.. These results indicate that a simple mechanism of independently diffusing surface vacancies and adatoms can lead to more rapid development of large 2-dimensional islands than by thermal annealing alone. Creation of multiple defects per ion, however, leads to continuous nucleation of new clusters which offsets the increased coarsening of the islands.

\section{Acknowledgements}

We gratefully acknowledge useful discussions and support from Jerry Floro, Tom Mayer and John Hunter. This work was performed at Sandia National Laboratories and supported by the U.S. Department of Energy under contract DE-AC04-94AL85000.

\section{References}

[1]. E. Chason, P. Bedrossian, K.M. Horn, J.Y. Tsao and S.T. Picraux, Appl. Phys. Lett. $57(1990) 1793$.

[2]. J. Mirecki Millunchik and S.A. Barnett, Appl. Phys. Lett. 65 (1994) 1136.

[3]. C-H. Choi, R. Ai and S.A. Barnett, Phys. Rev. Lett. 67 (1991) 2826.

J.A. Floro, B.K. Kellerman, E. Chason, S.T. Picraux, D.K. Brice and K.M. Horn, J. Appl. Phys. 77 (1995) 2351.

E. Chason, K.M. Horn, J.Y. Tsao and S.T. Picraux, Mat. Res. Soc. Symp. Proc. 128 (1989) 35. 
P. Bedrossian, J.E. Houston, J.Y. Tsao, E. Chason and S.T. Picraux, Phys. Rev. Lett. 67 (1991) 124.

E. Chason, P. Bedrossian, J.E. Houston, J.Y. Tsao, B.W. Dodson and S.T. Picraux, Appl. Phys. Lett. 59 (1991) 3533.

B.K. Kellerman, E. Chason, J.A. Floro, S.T. Picraux and J.M .White, Appl. Phys. Lett. 67 (1995) 1703.

\section{Figure Captions}

Figure 1. Surfaces produced by computer simulation of growth and ion bombardment. Lighter shades represent higher spots on the surface. (a) after initial growth of $0.25 \mathrm{ML}$ before coarsening, (b) after $77.5 \mathrm{~s}$ of thermal coarsening (no growth and no ion flux), (c) after $77.5 \mathrm{~s}$ of ion assisted coarsening, 1 vacancy per ion, ion flux equal to growth flux, (d) after $77.5 \mathrm{~s}$ of ion assisted coarsening, 9 defects ( 5 vacancies and 4 adatoms) per ion, ion flux equal to growth flux.

Figure 2. Island size, $n$, versus fraction of surface covered by islands of size $n$. (a)-(c) thermal coarsening, (no growth and no ion flux); (d)-(f) ion assisted coarsening, 1 vacancy per ion, ion flux equal to growth flux; (g)-(i) ion assisted coarsening, 9 defects (5 vacancies and 4 adatoms) per ion, ion flux equal to growth flux. Length of simulated time after initial growth sequence indicated in figure.

Figure 3. Evolution of mean of weighted island size distributions for thermal coarsening and ion assisted coarsening with 1 vacancy/ion and 9 defects/ion. 
Eric's Intensity Graph4.vi

Last modified on 8/19/96 at 1:30 PM

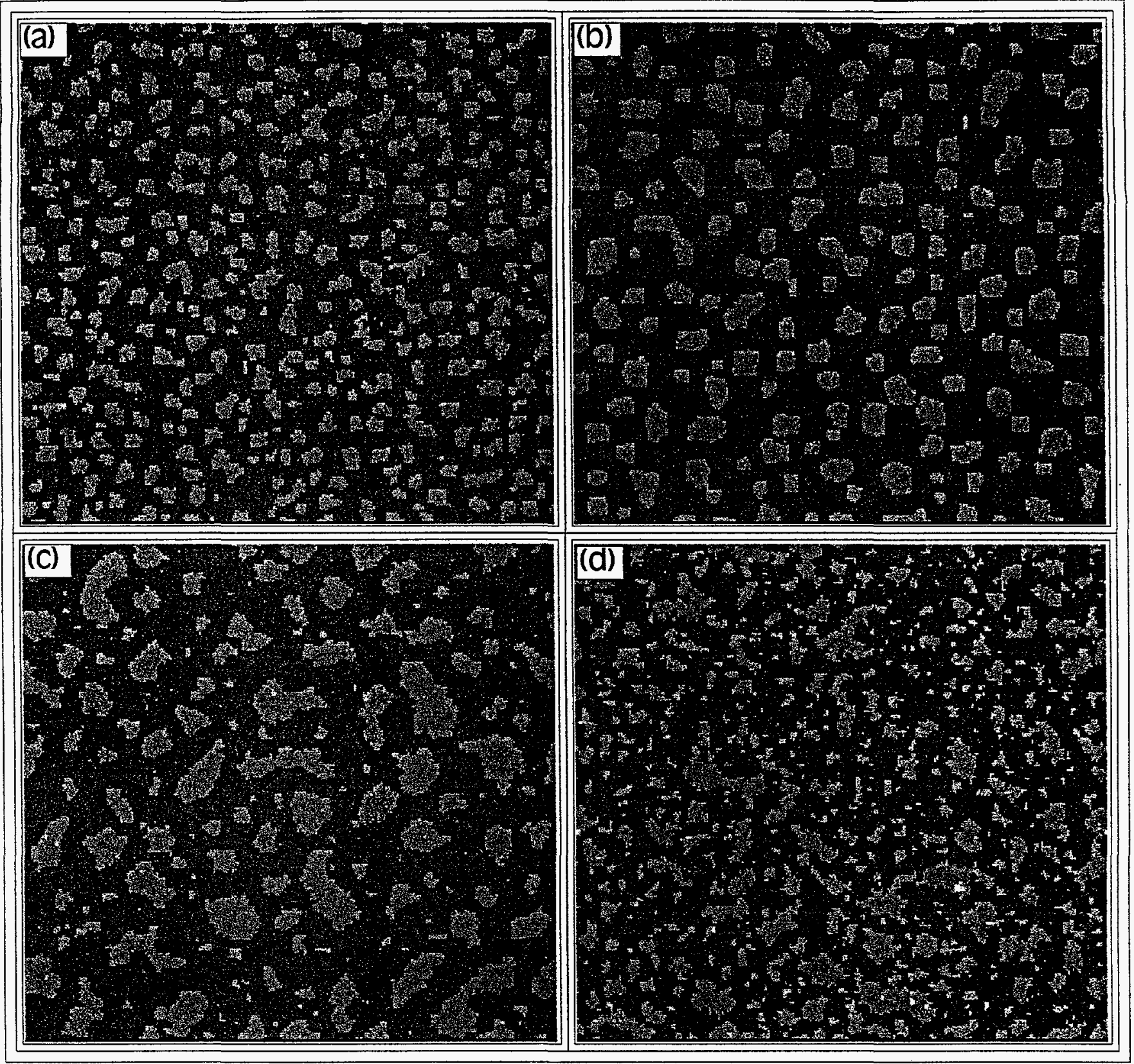



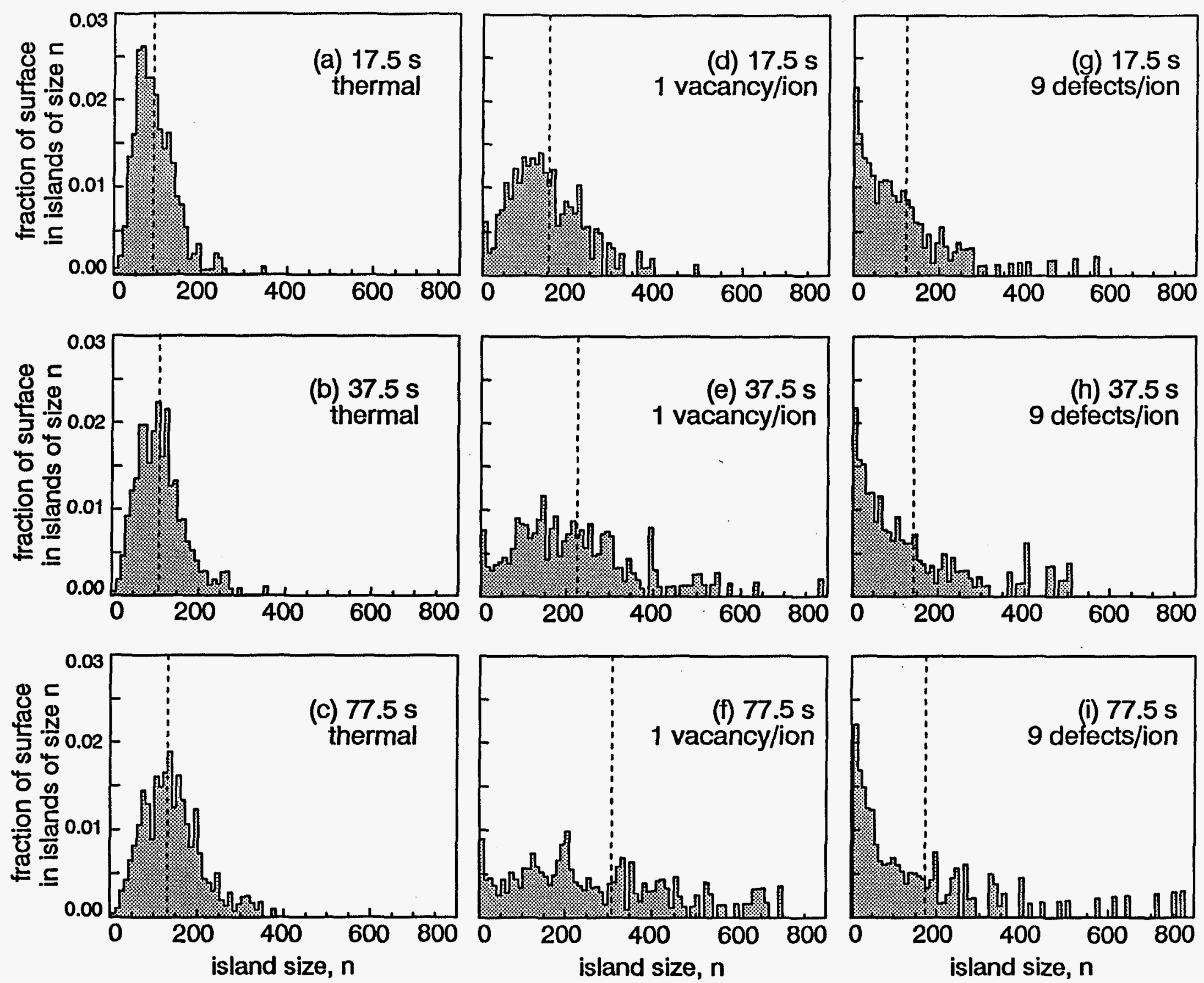


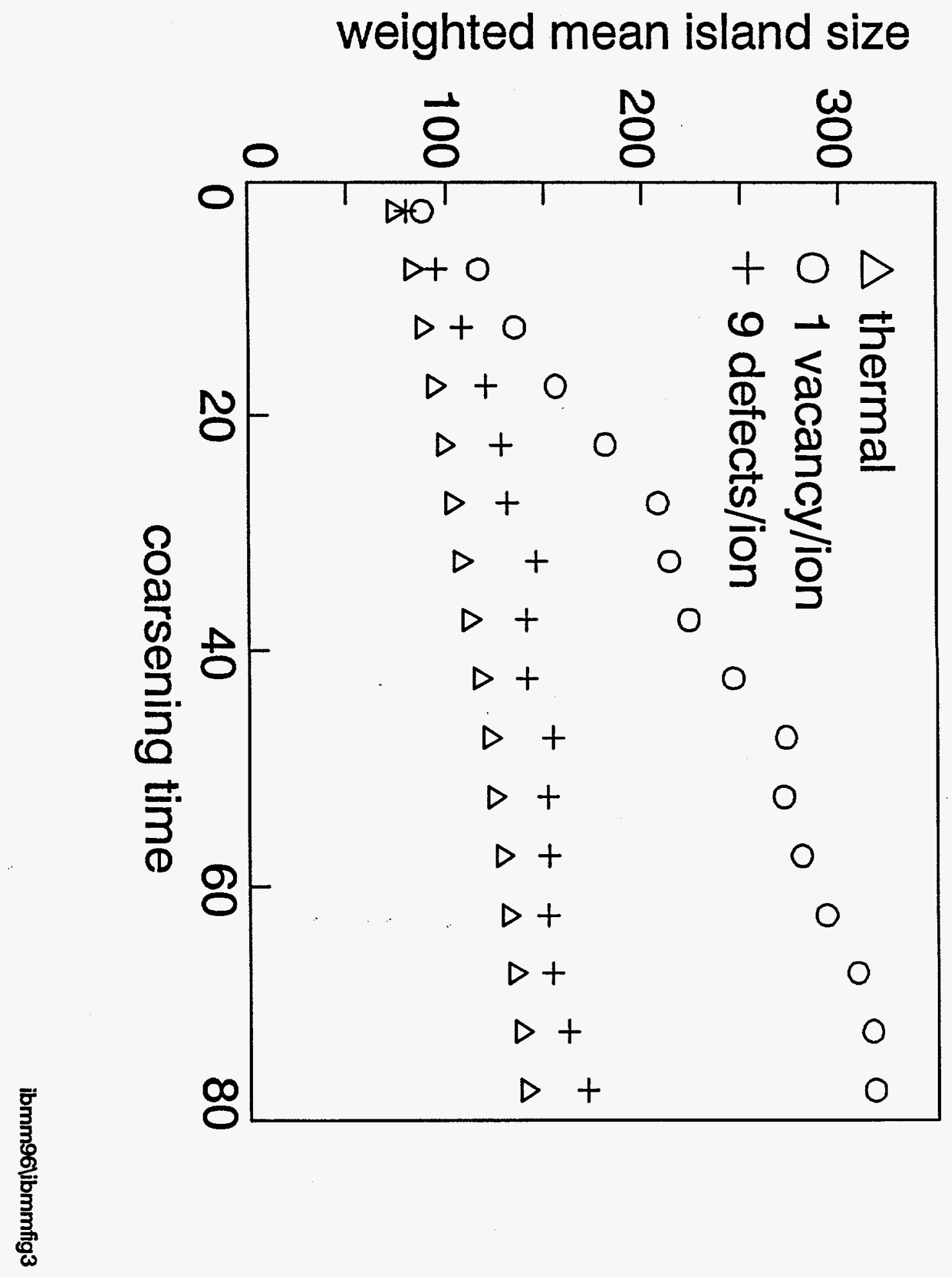

